\title{
Plagues in the ICU: \\ A Brief History of \\ Community-Acquired \\ Epidemic and Endemic \\ Transmissible \\ Infections Leading \\ to Intensive Care \\ Admission
}

\section{R. Bruce Light, MD}

\section{KEYWORDS}

- Infectious diseases in the ICU

- History of intensive care medicine

- Epidemic infectious diseases

From its very inception Intensive Care Medicine has been plagued by plagues. Indeed, infection is such a common and diverse theme in everyday ICU work that any article about the history of the problem must be somewhat selective. This article highlights eight different infection syndromes acquired in the community, that is, primarily transmissible from loci in the environment or from other people. All are serious infections frequently leading to ICU admission for shock, respiratory failure, or both. The short historical vignettes in this article are aimed at giving younger members of our specialty, who will not have personal memories of all of these events (as, unfortunately, some of us do) some sense of the variety and difficulty of the challenges posed by new infectious diseases, and examples of how, in each case, the scientific and critical care communities rose to those challenges. Each documented outbreak of disease was a surprise (and often a mystery) and the source of much public consternation, and each has taught us valuable lessons in improving the diagnosis, treatment, and prevention of infectious disease. 


\section{POLIOMYELITIS}

Intensive care, the epitome of the application of modern technology to medicine, arguably began as a response to the increasing numbers of older children and young adults developing paralytic polio during the early 1950s. Severe paralytic polio itself turns out to have been among the products of increasing modernity in the Western world.

Clinical recognition of the syndromes associated with the polio virus date back about 200 years. The fact that the disease was caused by a transmissible viral particle was demonstrated by 1908 . Until early in the twentieth century the virus was readily transmitted throughout the population almost continuously by personal contact and by the fecal-oral route via water. The result was that most people's first exposure to the virus occurred in infancy. At this age most infections resulted in a clinically unapparent infection, in part due to partial protection by maternal antibody, after which lifelong immunity was established. Only a few suffered a paralytic episode with the infection, at the time termed "infantile paralysis."

As hygienic standards of the advanced economy nations rose throughout the first half of the century, early childhood exposure to the virus declined. An increasing fraction of the population had their first exposure in late childhood or during young adulthood. In these age groups the likelihood that the infection will cause a paralytic syndrome is greatly increased, so both the incidence of clinically recognized infection and that of paralysis rose. By the early 1950 s clinically recognized cases had reached 15-25 annually for every 100,000 people in the United States, making it a major public health concern and a source of a great deal of fear in the general populace. ${ }^{2}$

More than $90 \%$ of polio virus infections are asymptomatic. However, at the more severe end of the clinical spectrum are the paralytic syndromes which range from paralysis of one or more limbs ("spinal polio") to syndromes with respiratory muscle or bulbar paralysis ("respiratory polio" or "bulbospinal polio") with loss of respiratory or upper airway muscle function or both. These more severe outcomes rise in incidence from about $0.1 \%$ in infants to more than $1 \%$ in older children and adults.

In the early part of the century development of polio with bulbar involvement was associated with a death rate of greater than $90 \%$, generally from respiratory failure. Use of a mechanical respirator to try to avert death was first attempted at the Children's Hospital in Boston in 1928, using an "iron lung." 3 The machine was basically a sealed box with a hole at one end for the patient's head to protrude, attached to two vacuum cleaners. The motors were then cycled to alternately create vacuum inside the box, expanding the patient's chest and causing the patient to inhale through the mouth (outside the box), then allowing air back into the box to permit exhalation. The design was further improved in 1931, and the machines came into increasingly broad use throughout North America and Europe during the severe outbreaks of the 1930s. Adoption of this therapy resulted in a significant reduction in mortality during these years.

Iron lungs, however, were cumbersome, difficult to use when trying to provide nursing care, and expensive. A more cost-effective and user-friendly approach to providing respiratory support was clearly needed. This finally came by bringing the positive pressure ventilation (PPV) concept out of the operating room. PPV was first used for respiratory support for polio victims at Blegdam Hospital in Copenhagen, Denmark, an innovation attributable to Danish anesthetist Bjorn Ibsen. ${ }^{4}$ During this large outbreak in 1952, some 200 medical students were put to work hand-ventilating dozens of patients through tracheostomies until the worst of the paralytic phase of the illness had passed, often several weeks. The concept quickly spread elsewhere and 
was widely adopted, yielding substantial reductions in mortality. For reasons of efficiency and convenience, patients needing respiratory support were often grouped in a single location where the necessary expertise and equipment were available. The introduction of PPV into a defined area of the hospital used to support respiratory failure was the genesis of the modern ICU and represents a signal event in the development of the field of Critical Care Medicine.

The introduction of effective vaccines, the Salk (inactivated) vaccine in 1952 and later the Sabin (live attenuated oral) vaccine in 1962, immediately and dramatically reduced the incidence of polio to less than one per 100,000 population by the early 1960 s and the incidence continued to fall thereafter. The last case of wild strain polio in North America was reported in 1979, and since that time the only cases of paralytic polio have been rare instances of disease due to variants of the live oral vaccine strain.

Polio does, however, still contribute to illnesses that may require intensive care in the form of the "post-polio syndrome." This occurs in patients who survived paralytic polio decades ago and who, over the years, develop a gradual decline in function in the originally affected nerves and muscles which can years later once again threaten them with disability and, in some cases, respiratory failure requiring intensive care. ${ }^{5}$

\section{LEGIONNAIRE'S DISEASE}

In July 1976, American Legion members attending a convention at a hotel in Philadelphia suddenly began falling ill with an acute febrile illness with pneumonia, often associated with extrapulmonary symptoms such as myalgia or diarrhea. ${ }^{6}$ Many developed acute respiratory failure requiring mechanical ventilatory support in ICU. Over 200 were affected and 34 died, an alarming mortality rate, especially since physicians caring for the patients had no idea what was causing the illness. Conventional microbiologic investigations yielded no convincing pathogens despite intensive investigation for the usual bacteria and viruses and other potential pathogens. Epidemiologic and various biologic investigations were quickly implemented by local health authorities and by the Centers for Disease Control and Prevention (CDC). These showed that the disease was likely airborne and that it occurred more frequently in older individuals who had underlying lung disease, smoked, or were relatively immunocompromised. Analysis of the likelihood of death revealed that those who received tetracyclines or macrolide antibiotics were more likely to survive than those who received only betalactams. However, no causal agent was uncovered, though many potential causes were excluded-most known bacteria and viruses, many biologic toxins and many environmental agents such as toxic chemicals and metals.

In 1977, Joseph McDade and Charles Shepard of the CDC reported the isolation of a novel fastidious gram-negative bacillus from the available clinical specimens. ${ }^{7}$ They named it Legionella pneumophila. This discovery was quickly followed by an explosion of knowledge about the organism and its ecology, antimicrobial susceptibility, and of other bacteria within the genus. Over time, demonstration that it was a widely occurring colonizer of brackish water, particularly in air conditioners, cooling towers, and water heaters and pipes, led to the implication of these reservoirs in several hundred outbreaks of the disease worldwide, especially in hospitals and other public health institutions, and hotels. Thus began widespread development of regulations and guidelines for limiting the degree of colonization of these water sources by Legionella, resulting in a reduction in the size, number, and scope of subsequent outbreaks.

Since the initial description of the disease, Legionella has, of course, been shown to be one of the major causes of community-acquired pneumonia (CAP), particularly in the more severe subset requiring ICU care; this fact underlies the major lesson from 
the outbreak - the nearly universal recommendation for inclusion of antimicrobial therapy for Legionell spp in any treatment regimen for severe CAP without another obvious cause. Although we now know that no amount of continuing effort can completely eliminate this organism from our environment, and that we will continue to see endemic cases, we also know that clusters of cases should trigger an investigation into finding the point source of the outbreak, a situation that continues to occur several times a year somewhere in the world.

In addition, the 1976 Philadelphia outbreak that defined "Legionnaire's Disease" was the first in the modern ICU era to demonstrate that major unknown infectious disease syndromes of severe consequence still exist, presaging the new infectious disease syndromes to be discovered in the years that followed.

\section{STAPHYLOCOCCALTOXIC SHOCK SYNDROME}

In the late 1970s, emergency rooms and ICUs throughout North America began to see an increasing number of young menstruating women presenting with a previously little-known syndrome characterized by sudden onset of a high fever, often associated with vomiting and diarrhea, quickly followed by severe hypotension. Early in the course most patients developed a diffuse macular rash, often with mucous membrane inflammation, with subsequent desquamation during convalescence. Patients frequently required massive fluid resuscitation because of systemic capillary leak, as well as vasopressor support, mechanical ventilation for adult respiratory distress syndrome, and even renal replacement therapy for acute renal failure, complicating the shock episode. One of the early clusters of observed cases was reported in 1978, and the term "toxic shock syndrome" was coined based on the isolation of an exotoxin-producing Staphylococcus aureus from mucosal surfaces or the site of a minor infection in the absence of bacteremia. ${ }^{8}$

As the case numbers rapidly increased case definitions for the syndrome were formulated and epidemiologic studies mounted. By 1980, over 1000 cases had been formally reported to the $\mathrm{CDC}$. $^{9}$ The case fatality rate was reported to be as high as $15 \%$ in the more severe cases included in the earliest reports, falling to about $3 \%-5 \%$ as recognition of the syndrome improved. By this time there were clear epidemiologic links between the syndrome and menstrual use of high-absorbency tampons which were often left in place longer than less absorbent products. Colonization of the tampon with Staphylococcus aureus was also implicated, consistent with the postulated toxin-mediated disease mechanism. ${ }^{10}$ Within months of these revelations the main manufacturer of the implicated tampons withdrew them from the market and women began changing tampons with greater frequency or stopped using them at the urging of public health authorities. The incidence of the syndrome immediately began to fall and within a few years, with the changing of use patterns of tampons and changes in their manufacture, toxic shock syndrome disappeared, for the most part, but not entirely, from the ICU.

Even at the height of TSS incidence in the United States, about $6 \%$ of the cases reported were nonmenstrual and $4 \%$ were in males. ${ }^{11}$ Subsequent development of the knowledge that the clinical syndrome was due to strains of Staphylococcus aureus that secrete a particular toxin (Toxic shock syndrome toxin 1, or TSST-1), which is both absorbable from mucosal surfaces and capable of producing a profound shock syndrome even in the absence of significant invasive infection, soon led to the more widespread recognition of the nonmenstrual toxic shock syndrome. This syndrome, which was almost certainly extant before but little-recognized, was perhaps the main lesson from the outbreak: even trivial staphylococcal skin or wound infections 
or mucosal surface colonization in the presence of a foreign body such as a nasal pack for nosebleed can lead to a severe shock syndrome if the organism is present and produces this toxin or one of several related ones. The recognition of the staphylococcal toxic shock syndrome also led to increasing understanding of the role of "superantigens" as a mechanism of disease-bacterial toxins capable of activating a large fraction (up to $20 \%$ ) of the total T-lymphocyte population. Such superantigens have since been implicated in a number of other disease syndromes, among them the streptococcal toxic shock syndrome (see below).

Over the past two decades, the incidence of menstrual and nonmenstrual staphylococcal TSS has been about one per 100,000 population in most areas. Busy ICUs will, therefore, continue to see occasional cases. However, there is some recent evidence that case numbers may be on the rise again in at least some areas, possibly because of a resurgence in the prevalence of toxin-producing strains in the community. ${ }^{12}$

\section{PNEUMOCYSTIS JIROVICII (FORMERLY CARINII)}

In 1980, physicians working in infectious diseases and critical care medicine thought they knew all about Pneumocystosis. The organism, then thought to be a protozoon, had been first described in 1909 by Carlos Chagas in Brazil, and since then had been clearly implicated as a cause of interstitial pneumonia in debilitated and malnourished children (in the aftermath of World War II) and, later, a cause of severe opportunistic pneumonia in immunocompromised patients, usually those being treated with highdose corticosteroids for connective tissue diseases or lymphoreticular neoplasms. ${ }^{13}$ In these patients it caused an impressively aggressive bilateral pneumonia leading to acute respiratory failure. This pneumonia was notoriously difficult to definitively diagnose, requiring bronchoscopy or open lung biopsy to demonstrate the small numbers of characteristic Pneumocystis organisms on special silver stains of clinical specimens. The mainstay of treatment at that time was pentamidine, generally given intramuscularly, giving way to trimethoprim/sulfamethoxazole after the publication in 1978 of a randomized clinical trial showing that it was at least as effective and generally better tolerated. ${ }^{14}$

In the early 1980s, a new form of the infection began to be seen with regularity. Young men began to present to hospital with a rather more indolent diffuse bilateral pneumonia that nevertheless went on to cause respiratory failure and which, when investigated, proved to be due to Pneumocystis. ${ }^{15}$ The course of the disease was quite different from what physicians had been used to up to then. It began more gradually, progressed at a slower pace and was associated with a much lesser systemic inflammatory response. Microscopy of respiratory specimens revealed exponentially more organisms than previously seen, such that many patients could be diagnosed from sputum specimens rather than bronchoscopy, and biopsy was virtually never needed. Nobody had any idea why this was happening, although it quickly became apparent that the underlying cause of the infection was a new form of severe deficiency of cell-mediated immunity.

Epidemiologic investigations were soon underway. Patterns began to emerge. Many of the young men were Haitian or had been to Haiti. Many were homosexual, bisexual, or had worked in the sex trade; others had abused intravenous drugs. The many fewer women with the disease had similar exposures. Theories proliferated. Was it immunologic exhaustion from exposure to too many microbial stresses? Toxins? Drugs used in the sex trade? Multiple and synergistic viral infections?

Through the early 1980s, the case load grew. ICUs throughout North America and, later, in Europe and elsewhere, saw increasing numbers of young people, mainly men, 
with severe respiratory failure due to Pneumocystis pneumonia. By 1981 they were being called patients with acquired immunodeficiency syndrome (AIDS) on the basis of demonstration of low numbers of CD4 lymphocytes in the blood, but the cause remained unclear. Then, in 1984, Montagnier and Barre-Sinoussi at the Pasteur Institute in France isolated a viral pathogen that they named Lymphadenopathy-Associated Virus (LAV). At the National Institutes of Health in the United States, Gallo demonstrated that the virus (which he referred to as Human T-cell Leukemia Virus III, based on an unproven relationship to other viruses he had previously discovered) definitively caused AIDS. The virus, now called human immunodeficiency virus (HIV)-1 was isolated and described and the first diagnostic kits devised, resolving the mystery of causation. Montagnier and Barre-Sinoussi would eventually win the Nobel Prize in Medicine in 2008 for their contribution.

During the 1980s, patients continued to present with severe pneumonia requiring respiratory support and intensive antimicrobial therapy, often with less than satisfactory results. As knowledge progressed, HIV-associated Pneumocystis infection in the ICU changed its face several times over the years. At the beginning of the epidemic, most patients presenting for care with HIV/AIDS and Pneumocystosis were severely ill with diffuse pneumonia and hypoxemic respiratory failure and many died, $80 \%-90 \%$ in most centers, prompting widespread debate about whether such patients should even be admitted to ICU for mechanical ventilatory support. However, as experience with the disease developed it became clear that an early and aggressive approach could improve prognosis. It was found that in the AIDS population even minor respiratory symptoms with few or no abnormalities on chest radiograph could be due to Pneumocystis infection in the earlier stages, and that even modest degrees of arterial oxygen desaturation signaled impending respiratory failure. Earlier bronchoscopy for diagnosis followed by prompt antimicrobial therapy, with pentamidine predominantly in the early 80 s and later primarily with trimethoprim/sulfamethoxazole, led to overall mortality rates falling to the $50 \%-60 \%$ range by the middle of the decade.

The advent of systemic corticosteroids therapy for early respiratory failure in AIDSassociated Pneumocystosis was then shown to further reduce the numbers of patients progressing to advanced respiratory failure, leading to reductions in the numbers of cases needing ICU admission and further reducing overall mortality rates to the $15 \%-20 \%$ range. ${ }^{16}$ But for patients requiring ICU care mortality rates were as high as before the use of steroids and often higher, likely related to the fact that most patients developing respiratory failure had already failed to improve or had progressed despite intensive antimicrobial and corticosteroid therapy. ${ }^{17}$

Along with these developments in management of the disease, progress was being made on HIV itself. Following identification of the virus in 1984, there soon followed increasingly reliable diagnostic tests for the infection, leading to earlier identification of cases and monitoring of CD4 lymphocyte counts. By the early 1990s, studies supporting widespread use of chemoprophylaxis against Pneumocystis in all patients with CD4 counts $<200 / \mathrm{mm}^{3}$ were available and became standard public health agency recommendations. Pneumocystosis, which in the 1980s and 1990s was one of the principal causes of hypoxemic respiratory failure in many ICUs in North America and western Europe, began to decline rapidly in incidence, becoming relatively uncommon even before the widespread adoption of highly active antiretroviral therapy, which has, since the mid-1990s, caused the disease to all but disappear from our ICUs.

Although many lessons can be drawn from the battle against AIDS-related pneumoncystis pneumonia during the 1980s and 1990s, for ICU and infectious diseases practitioners one of the main ones comes from the sad fact that once patients had 
developed full-blown hypoxemic respiratory failure even the best intensive care could only deliver $20 \%$ survival rates. The really large gains in survival came not from better ICU technology but from pre-empting the disease on multiple fronts, including earlier diagnosis of HIV infection, early diagnosis and antimicrobial treatment of pneumonia, steroid treatment of early respiratory failure, antimicrobial prophylactic regimens and, later, effective antiretroviral therapy.

\section{HANTAVIRUS PULMONARY SYNDROME}

Until 1993, the only members of the Bunyaviridae family of viruses known to cause disease in North America were members of the genus Bunyavirus, all causing mosquito-borne viral encephalitis, mainly in children (California encephalitis). Other members of the family were known to cause serious febrile illnesses, encephalitides and hemorrhagic-fevers in Africa and Asia (Rift Valley Fever, Crimean-Congo Hemorrhagic Fever, Hemorrhagic Fever with Renal Syndrome). However, in the spring of 1993 wetter-than-usual conditions in the American Southwest led to increased availability of food for deer mice, leading to a population explosion and increasing movement of rodents into human-occupied spaces, increasing the chance that humans might be exposed to the rodents and their excreta.

In rapid succession, several previously healthy young people, mainly Navajos, presented to health care institutions in the Four Corners area of the Southwestern United States, all with fulminant illnesses leading to shock and acute respiratory failure requiring ICU care. By early June that year, 24 cases had been identified and 12 had died..$^{18}$ In most cases the illness had started with fever and widespread myalgia, soon followed by cough, then by cardiovascular collapse due to a severe systemic capillary leak syndrome and by acute respiratory failure due to low-pressure pulmonary edema. In some cases the time from onset to ICU or death was as little as 24 hours, in others a few days. ${ }^{19}$

Remarkably, although no pathogen was initially identified from blood or tissues, in less than a month after the first report of a possible outbreak, serologic testing had demonstrated antibody cross-reactivity with a range of known pathogens of the Hantavirus group, suggesting that the disease was due to a previously unknown member of this group. Shortly thereafter exposure to deer mice and their excreta was implicated as the likely source of the infection.

The mortality rate for the early cases of hantavirus pulmonary syndrome (HPS) was extremely high $-80 \%$ in the initially reported group of patients-mostly due to intractable shock and unsupportable hypoxemic respiratory failure due to acute respiratory distress syndrome (ARDS). However, this improved with clinical experience as it became evident that administration of large amounts of intravenous fluids in the face of profound capillary leak only resulted in much worse generalized and pulmonary edema, with little improvement in the shock state and only worsening of the respiratory failure. Management changed to an approach limiting the amount of fluid administered early in the course together with earlier institution of inotropic support, resulting in a much improved survival rate of about $60 \%$, generally with minimal or no long-term sequelae in survivors. ${ }^{20}$

In subsequent years development of increasingly specific serologic and virologic testing has demonstrated that this disease had been present but unrecognized throughout North and South America long before this outbreak, and that there are several related viruses, each associated with a particular rodent, causing endemic disease and the occasional outbreak. By the mid-1990s, over 150 cases were reported in 25 states, mainly in the Southwest, and cases have since been reported 
in small numbers in most other states, Canada, Mexico, and South America, where several outbreaks have occurred. Whereas occasional cases continue to be seen in ICUs in all these areas, no further major outbreaks have yet occurred in the United States or Canada, though clearly remaining a threat under the right conditions; the only currently available preventive measure is avoiding rodent contact. ${ }^{21}$

Steven Simpson, MD, one of the intensivists at the Health Sciences Center in Albuquerque, New Mexico, who was closely involved in the initial Four Corners outbreak, points out that the event highlights several trends in subsequent disease outbreaks in North America. One is the extreme rapidity with which novel pathogens and potential pharmacotherapeutic agents can now be identified. Whereas the pathogen in the Legionnaire's outbreak took almost a year to identify, researchers identified the HPS pathogen and its source in just months. Computerized access to data and data analysis along with virtually instantaneous electronic transmission of information plays a central role in this development.

The initial HPS outbreak has several ICU-related lessons to teach us. While the aforementioned treatment strategies effective in a systemic capillary leak syndrome have been absorbed by the critical care community, it appears that one lesson taken to heart by the local ICU teams failed to disseminate to the broader ICU community. The initial outbreak was accompanied by a marked element of fear and concern among health care workers, including those in the ICU, and a significant level of panic in the local community; a combination of this fear, the requirement for rigorous quarantine precautions, and a marked increase in transfers to the ICU of any severely ill patients with symptoms remotely compatible with HPS resulted in some compromise of ICU operations, due to being completely overwhelmed. This might potentially have been avoided by an awareness that for an effective epidemic response, it is essential to include both hospital and ICU operations in each locale.

The outbreak also reinforces the principle that nearly all old and most new epidemic infectious diseases have their origin in close contact between humans and other species of animal, both wild and domestic, and new kinds and quantities of such contact are likely to cause new, or newly recognized, disease syndromes.

\section{STREPTOCOCCALTOXIC SHOCK SYNDROME AND NECROTIZING FASCIITIS}

Streptococcus pyogenes was one of the first bacteria ever to be conclusively linked to human disease (puerperal infection associated with childbirth). However, over the past 125 years the nature of the diseases stemming from it has changed dramatically on several occasions. At the turn of the last century, it was well known as a cause of streptococcal pharyngitis, erysipelas, and wound infections. It also caused severe septicemic illnesses that frequently led to death. Osler ${ }^{22}$ knew Streptococcus pyogenes as a principal cause of thoracic empyema following pneumonia or severe cases of scarlet fever, and also as a major cause of primary bacteremia with sepsis. These more severe manifestations of streptococcal infection became increasingly uncommon as the twentieth century progressed, particularly after the arrival of antibiotics mid-century.

Notably, Osler did not mention Streptococcus as a cause of necrotizing fasciitis or being associated with soft tissue necrosis in wound infections. This syndrome was first described by Meleney in $1924,{ }^{23}$ at that time, it was characteristically a slowly evolving gangrenous infection, usually of surgical wounds, which often responded well to debridement and was associated with a mortality rate of only $20 \% .^{24}$

For over a generation after the advent of the modern antibiotic era, Streptococcus pyogenes was seldom a problem that led to critical illness-soft tissue infections and 
the occasional bacteremia were generally very amenable to treatment; extensive surgery or drainage was seldom required, and cases requiring ICU support for shock or respiratory failure were rare. Beginning in the mid-1980s, medical practitioners in centers across North America and Europe began seeing previously unknown forms of severe streptococcal disease, soon labeled Streptococcal necrotizing fasciitis and Streptococcal toxic shock syndrome. ${ }^{25}$

Streptococcal toxic shock syndrome (Strep TSS) is any infection with Streptococcus pyogenes that is associated with a rapidly progressing systemic toxic response characterized by early onset of high fever and myalgia, often with prominent gastrointestinal symptoms, and by rapid progression to hypotension and multiple organ system failure. The illness usually requires ICU support for massive fluid resuscitation, vasopressor and inotropic support and mechanical ventilation. Although some cases have primary bacteremia, many others have a localized focus of infection, most often in soft tissues, that only becomes clinically apparent after the onset of shock.

Streptococcal necrotizing fasciitis is often associated with Strep TSS and, as mentioned, is often only correctly diagnosed after the onset of shock. The most characteristic story is presentation to a physician or an emergency room with abrupt onset of severe pain, often in an extremity with minimal or no evidence of cutaneous injury. At this stage severe systemic toxicity is usually not present and, since examination of the painful site is also at this stage quite unremarkable, patients are frequently sent home with analgesics and reassurance. Over the next 4-48 hours pain at the site of infection continues to increase, soft tissue swelling and redness appear above the deeper tissues that are undergoing ongoing necrotizing infection, eventually resulting in full-thickness necrosis evidenced by ecchymosis, cutaneous necrosis, and bullae formation. ${ }^{26}$ Early or later in this course Strep TSS frequently occurs.

When these cases first began to appear, clinicians' approach to both the sepsis and the tissue necrosis was essentially the same as that used for apparently similar syndromes caused by other bacteria. A broad spectrum antimicrobial was started, fluid resuscitation begun and imaging studies ordered to better define the source of infection causing pain or localized swelling. Imaging frequently demonstrated only soft tissue swelling consistent with cellulitis, so surgery was often deferred until superficial signs of tissue necrosis became obvious, and then when surgery was done it was often performed using the conventional approach of trying to conserve as much tissue as possible. The result was that treatment was often too little and too late, with mortality rates exceeding $70 \%$ in many reported series.

With the realization that treatment, to be successful, must be swift and aggressive, approaches to therapy changed. Emergency physicians were increasingly alerted to the fact that severe pain at any body site, even with relatively minimal localized physical findings and particularly if accompanied by signs of systemic inflammation, could represent necrotizing fasciitis. Surgeons began to be consulted much earlier, and any localized pain with swelling more often led to diagnostic surgical exploration rather than imaging and waiting. Antimicrobial strategies changed. Addition of clindamycin to the usual penicillin or other beta-lactam therapy was advocated and widely adopted, based on results from animal models of the syndrome and on pharmacologic and physiologic considerations, including its ability to inhibit bacterial protein (ie, toxin) synthesis, penetrate necrotic tissues, and inhibit inflammatory cytokine synthesis. ${ }^{27}$ Toxin neutralization using pooled intravenous gamma globulin was also advocated with the support of primarily historical case-control studies. ${ }^{28}$ In most centers, implementation of these approaches has led to dramatic reductions in mortality rates to about $20 \%-30 \%$ although, in the absence of any adequate controlled trials, it remains unclear what the relative contribution of each of these measures has been to the improved outcome. 
Unlike several of the other "plagues" discussed above, this is one that is still very much with us. The Streptococcus pyogenes strains most strongly associated with severe invasive disease (M-protein types 1 and 3 ) have increasingly been supplanting those associated with less severe disease resulting in an endemic sporadic case-rate for severe disease of one to 20 cases $/ 100,000$ population yearly, with intermittent larger-scale community outbreaks, both of which will continue to require vigilance and an aggressive therapeutic stance from the critical care community. ${ }^{29}$

\section{SEVERE ACUTE RESPIRATORY SYNDROME}

The first case of this apparently novel severe viral respiratory infection occurred in Guangdong Province in southern China in November 2002. The victim, a farmer, died of an undiagnosed "atypical pneumonia." Over the ensuing weeks several more cases of severe respiratory syndromes began to appear in the region, also undiagnosed. By the end of November there had been enough such cases to generate considerable alarm among the medical community in China, generating Internet communications between institutions which were picked up by international monitoring agencies. This led to a request from the World Health Organization (WHO) for information about the outbreak, but no information was forthcoming from Chinese authorities. The first official report about the outbreak was made to public health authorities in Guangdong in early January 2003, with a later report to the WHO in February that, in retrospect, did not fully make clear either the nature or the scale of the problem. ${ }^{30}$

Transmission of the disease within China continued to occur, leading to rapidly increasing numbers of cases in South China, then throughout the country and to the capitol Beijing (where one of the largest outbreaks occurred). Exposure of Chinese travelers and visitors to the country was inevitable, given the scale of the outbreak. One exposed individual was a physician from mainland China who, incubating the disease during his travel, stayed at the Metropole hotel in Hong Kong in early March. Later investigations showed that he transmitted the virus to at least 16 other guests at the hotel, who then carried it by international air travel to Taiwan, Singapore, Vietnam, and Canada.

One of these contact cases was an American businessman headed for Singapore. Becoming ill while in transit, he stopped in Hanoi where he was admitted to hospital with a severe pneumonia, to which he eventually succumbed. Soon after, a number of health care workers who had been in contact with him also became acutely ill. Fortunately for the course of the outbreak, one of the consultants on the case was an Italian physician working with the WHO in Vietnam, Dr. Carlo Urbani. He immediately recognized that this was a previously unknown severe atypical pneumonia that was relatively easily transmissible and reported it to the $\mathrm{WHO}$; this led to immediate mobilization of investigative efforts and worldwide alerts about the threat. ${ }^{31}$ Unfortunately, in the course of caring for the victims of the disease in Hanoi, Dr. Urbani himself contracted the infection and died of it later.

As information from China became more available, it became clear that by this time there had already been hundreds of cases and numerous deaths. The majority of the initial wave of cases were noted to have occurred primarily in farmers and food handlers, particularly those working in food markets where live wild animals were kept and sold for food. The second large wave of those affected were health care workers exposed in hospital to patients with the disease. The illness was characterized by fever and myalgia with gastrointestinal symptoms in the initial phase, occurring an average of 5 days after exposure (range 2-10 days). Many cases got no worse than this, but others went on to develop dyspnea associated with radiographic evidence of a diffuse, patchy pneumonitis which, in some, progressed to ARDS. An average of 
$15 \%$ required mechanical ventilatory support, and when the data were all in from later phases of the outbreak, mortality rates averaged about $10 \%$ overall, worse in the aged and debilitated, lower in the young and healthy.

The largest outbreak outside Asia occurred in Toronto, Canada. The index case, a visitor to China, returned to Canada and died of pneumonia at home, undiagnosed, in early March 2003. Shortly thereafter, one of his sons was admitted to hospital with a severe respiratory illness and died a few days later. By this time, four other family members had become ill and had been admitted to hospital; the first cases of affected health care workers appeared soon after among those who had cared for the dying son of the index case. Within days, other instances of transmission from undiagnosed contacts of the initial cases in hospitals, doctors' offices, emergency rooms, and at social events were leading to admission of cases to several hospitals throughout Toronto. The response of the public health authorities, beginning soon after the WHO global alert and coincident with the recognition of the first local cases, was quick and vigorous, including closure of the main affected hospital, intensive follow-up of probable contacts, quarantine of suspected cases based on a fairly inclusive case definition and strict institution of barrier contact protection for health care workers. ${ }^{32}$ By mid-April the number of new cases was rapidly declining, although there was one cluster of late cases related to exposure of a large number of health care workers during the resuscitation and difficult intubation of a critically ill patient. A later cluster of cases also occurred in a rehabilitation hospital, where it appeared that unrecognized contacts from the first phase of the outbreak had been transferred and transmitted the disease to other patients and staff.

The worldwide outbreak was essentially over by July 2003 . There were a total of 8098 reported cases from 26 countries, with 774 deaths.

Intensive epidemiologic and laboratory study of the disease by investigators and laboratories worldwide led to unprecedented rapid growth in knowledge about the causative agent. The virus, more or less simultaneously characterized at a number of laboratories around the world, proved to be a previously unknown coronavirus (severe acute respiratory syndrome [SARS]-CoV) with capacity to infect and spread from a variety of wild animals to humans. Epidemiologic, serologic, and virologic evidence was developed linking human cases to exposure to infected wild animals, including masked palm civets, raccoon dogs, ferrets and ferret badgers, all being sold for human consumption in markets in China. ${ }^{33}$ Control of their transport and sale and exposures to humans by Chinese health authorities was probably one of the major factors in bringing the first outbreak under control, the partial failure of which later led to a second, much smaller outbreak late in 2004. Although the initial speculation was that one or more of these wild animals were the reservoir in nature for the infection, it now appears more likely that the viral reservoir is actually bats, with crosstransmission of the virus between bats, food animals, and humans in crowded markets leading to development of strains with the capacity to transmit between humans. ${ }^{34}$

Public health authorities worldwide learned much from SARS about the importance of effective international communication in developing a rapid and effective response to outbreaks of novel viruses, and more about how to go about containing such infections within communities and hospitals. Several intensivists involved in the outbreak credit e-mail communications from other international outbreak sites for effective advice on critical elements of disease protection (eg, Powered Air Purifying Respirators and full contact rather than droplet precautions) and therapy. For the critical care community, perhaps one main lesson was the importance of "super-spreading incidents" in propagating the disease in hospitals. Many of these occurred in critically 
ill patients undergoing resuscitation with difficult or traumatic intubation, generating aerosols in closed spaces which contained many superfluous and inadequately protected health care workers. Handling these situations safely depends crucially on identifying the potential risk and undertaking the resuscitation and intubation using the most experienced operators available, adequately protected with basic barrier precautions (eye protection, gloves and surgical face-masks), using sedation or paralysis as necessary to minimize trauma and aerosol generation, and with only essential and adequately protected staff in the room. ${ }^{35}$ This likely applies to many other situations with potential for disease transmission to health care workers.

Unfortunately, this epidemic again points out the primary lesson that was not absorbed from the earlier HPS outbreak, namely, the need for detailed preplanning and preparation for a major infectious disease epidemic that is inclusive of hospital and ICU operations in each locale. According to participants, the SARS outbreak demonstrated many of the same early ICU operational problems that plagued the HPS outbreak albeit on a larger scale.

\section{INFLUENZA}

In the ICU era, there has yet to occur a true influenza pandemic with a high attack rate in all age groups and associated high hospitalization and mortality rates, as was seen in the great 1918 pandemic. In that worldwide disaster, it is estimated that $30 \%$ of all people became ill with the virus and an estimated $50-100$ million died. ${ }^{36}$ Minor recent pandemics in 1968 and 1977 had less than one twentieth of the impact of the 1918 influenza, not greatly different from the yearly interpandemic influenza the world has been experiencing in the 30 years since.

Interpandemic influenza epidemics since 1977 have been caused primarily by H3N2 and $\mathrm{H} 1 \mathrm{~N} 1$ influenza viruses, to which most of the population has developed some degree of immunity from prior infection or vaccination. The result is what public health authorities have become used to seeing: each year a slightly different influenza $A$ appears in Asia with minor antigenic changes in the HA or NA surface proteins (termed drift), making it infectious once again for humans whose immune systems have yet to be exposed to the new variant, and a new epidemic is launched. When the "flu" arrives in an area, cases begin to appear suddenly and there is rapid spread in the population, usually with $20 \%-30 \%$ becoming infected over a 6 -week period with a peak in case numbers at week two or three. About half of those infected will seek medical attention, many more than once, and one to about 25 per thousand infected will be admitted to hospital with a respiratory syndrome such as pneumonia, chronic obstructive pulmonary disease exacerbation, asthmatic attack, or cardiac failure, the rate depending on age and underlying comorbidities. Overall, about $0.1 \%$ of those infected die, with mortality rates among those with major comorbidities up to $5 \%$. These latter cases constitute most of the increase in the ICU case load which most units experience every winter. The load is sometimes taxing but usually not overwhelming.

A true pandemic is unlikely to play out this way. How different it would be depends on a number of factors: the antigenic difference in the new Influenza virus compared with the old (ie, the antigenic "shift" to a different one of 15 HA or 9 NA protein subtypes due to introduction of a variant from another influenza-susceptible species), how transmissible the new virus is, how virulent it is, how susceptible it is to antiviral drugs, and whether the world is prepared for it with drug availability and vaccines.

The prototype severe pandemic was the Spanish influenza of 1918, an H1N1 virus. The most recent circulating influenza virus just before that time was an H3N8 that had arrived in 1901. Current evidence suggests that an avian influenza virus underwent a period of evolutionary adaptation, possibly in another susceptible species such as 
swine, fitting it for transmission to humans, which it then did. ${ }^{36,37}$ This H1N1 virus had not been previously experienced by any segment of the population except the very old, so nearly everyone, particularly non-elderly adults and children, was without immunity and was at risk of severe infection. Attack rates, as noted earlier, were extremely high everywhere as were rates of primary influenza pneumonia, complicating bacterial pneumonia, and death. In the United States, death rates were more than 20-fold higher than in any influenza pandemic since. An outbreak of influenza on this scale, if unchecked by effective antiviral therapy or vaccines, would render ICU care such as mechanical ventilatory support for respiratory failure irrelevant. Even today, with maximal respiratory support, most patients with diffuse primary viral pneumonia complicated by respiratory failure cannot be saved, and the numbers presenting for such care in a short space of time, if comparable to the 1918 pandemic, would overwhelm our current ICU capacity within days.

Currently the main apparent threat of a new pandemic comes in the form of the $\mathrm{H} 5 \mathrm{~N} 1$ influenza virus. This virus is now present nearly worldwide in migratory and, intermittently, domestic bird populations. From time to time, transmission of the virus from birds to humans occurs, generally from close contact situations. WHO data indicate that there have been 387 laboratory-confirmed cases of such transmission from 2003 to mid-2008. ${ }^{38}$ The mortality rate has exceeded $60 \%$, although it is likely that many less severe cases do not come to medical attention and are therefore not counted as confirmed case survivors. To date no instances of transmission to humans by humans or other mammals has been documented. However, the threat remains that if this virus were to become capable of human-to-human transmission by adaptation in another susceptible mammalian host such as swine, a pandemic on the order of the 1918 event could occur.

With no true pandemic for over 30 years, including all of the ICU era, health authorities worldwide are deeply engaged in trying to learn the lesson of this new "plague" before it actually occurs. It is clear that we will need excellent international communication, rapidly enactable containment and quarantine plans and, if possible, effective antivirals and vaccines to deal with the H5N1 virus. If it evolves as feared and becomes easily transmissible while retaining its current virulence; modern life-sustaining technology alone will be no shield at all.

The last 60 years have seen remarkable advances in the ability to diagnose and treat infectious diseases and handle infectious disease outbreaks. For the most part, the major plagues of antiquity remain historical footnotes. However, despite these advances, there is clear evidence that major pandemic illness is always just one outbreak away. In addition to the HIV pandemic, the smaller epidemic outbreaks of Legionnaire's disease, hantavirus pulmonary syndrome, and SARS, among many others, points out the potential risk associated with a lack of preplanning and preparedness. Although pandemic influenza is at the top of the list when discussing possible future major infectious disease outbreaks, the truth is that the identity of the next major pandemic pathogen cannot be predicted with any accuracy. We can only hope that general preparedness and the lessons learned from previous outbreaks suffice.

\section{REFERENCES}

1. Paul JR. A history of poliomyelitis. New Haven (CT): Yale University Press; 1971.

2. Oshinsky DM. Polio: an American story. New York: Oxford University Press; 2005.

3. Drinker P, Shaw LA. An apparatus for the prolonged administration of artificial respiration. J Clin Invest 1929;7:229-47. 
4. West JB. The physiologic challenges of the 1952 Copenhagen poliomyelitis epidemic and a renaissance in clinical respiratory physiology. J Appl Physiol 2005;99:424-32.

5. Trojan D, Cashman N. Post-poliomyelitis syndrome. Muscle Nerve 2005;31:6-19.

6. Fraser DW, Tsai TB, Orenstein W, et al. Legionnaire's disease: description of an epidemic of pneumonia. N Engl J Med 1977;297:1189-97.

7. McDade JE, Shepard CC, Fraser DW, et al. Legionnaire's disease: isolation of a bacterium and demonstration of its role in other respiratory diseases. $\mathrm{N}$ Engl J Med 1977;297:1197-203.

8. Todd J, Fishout M, Kapral F, et al. Toxic-shock syndrome associated with phagegroup-1 staphylococci. Lancet 1978;2(8100):1116-8.

9. Centers for Disease Control. Toxic-shock syndrome - United States. MMWR Morb Mortal Wkly Rep 1980;29:229-30.

10. Shands KN, Schmid GB, Dan BB, et al. Toxic-shock syndrome in menstruating women: association with tampon use and Staphylococcus aureus and clinical features in 52 cases. N Engl J Med 1980;303:1436-42.

11. Centers for Disease Control. Epidemiologic notes and reports, toxic-shock syndrome, United States. MMWR Morb Mortal Wkly Rep 1982;31:201-4.

12. Schlievert PM, Tripp TJ, Peterson ML. Reemergence of staphylococcal toxicshock syndrome in Minneapolis- St. Paul, Minnesota, during the 2000-2003 surveillance period. J Clin Microbiol 2004;42:2875-6.

13. Hughes WT, Feldman S, Aur RJ, et al. Intensity of immunosuppression and the incidence of Pneumocystis carinii pneumonia. Cancer 1975;36:2004-9.

14. Hughes WT, Feldman S, Chaudharg SC, et al. Comparison of pentamadine isothionate and trimethoprim/sulfamethoxazole in the treatment of Pneumocystis carinii pneumonia. J Pediatr 1978;92:285-91.

15. Centers for Disease Control. Pneumocystis pneumonia - Los Angeles. MMWR Morb Mortal Wkly Rep 1981;30:250-2.

16. Bozette SA, Sattler FR, Chiu J, et al. A controlled trial of early adjunctive treatment with corticosteroids for PCP in AIDS. N Engl J Med 1990;323:1451-7.

17. Azoulay E, Parrot A, Flahault HA, et al. AIDS-related pneumonia in the era of adjunctive steroids. Am J Respir Crit Care Med 1999;160:493-9.

18. Centers for Disease Control. Outbreak of acute illness - southwestern United States, 1993. MMWR Morb Mortal Wkly Rep 1993;42:421-4.

19. Duchin JS, Koster FT, Peters CJ, et al. Hantavirus pulmonary syndrome: a clinical description of 17 patients with a newly recognized disease. N Engl J Med 1994; 330:949-55.

20. Halin GW, Simpson SQ, Crowell RE, et al. Cardiopulmonary manifestations of Hantavirus pulmonary syndrome. Crit Care Med 1996;24:252-8.

21. Centers for Disease Control. Hantavirus pulmonary syndrome - United States: updated recommendations for risk reduction. MMWR Morb Mortal Wkly Rep 2002;51:1-12.

22. Osler W. The principles and practice of medicine. New York: D. Appleton and Company; 1892. p. 110-8.

23. Meleney FL. Hemolytic streptococcus gangrene. Arch Surg 1924;9:317-64.

24. Meleney FL. Hemolytic streptococcal gangrene: the importance of early diagnosis and operation. JAMA 1929;92:2009-12.

25. Cone LA, Woodward DR, Schlievert PM, et al. Clinical and bacteriological observations of a toxic-shock-like syndrome due to Streptococcus pyogenes. N Engl J Med 1987;317:146-9. 
26. Barker FG, Leppard BJ, Seal DV. Streptococcal necrotizing fasciitis: comparison between histological and clinical features. J Clin Pathol 1987;40:335-41.

27. Stevens DL, Bryant AE, Hackett SP. Antibiotic effects on bacterial viability, toxin production and host response. Clin Infect Dis 1995;20(Suppl 2):S154-7.

28. Kaul R, McGeer A, Norrby-Teglund A, et al. Intravenous immunoglobulin therapy for streptococcal toxic-shock syndrome - a comparative observational study. The Canadian Streptococcal Study Group. Clin Infect Dis 1999;28:800-7.

29. Johnson DR, Stevens DL, Kaplan EL. Epidemiologic analysis of group A streptococcal serotypes associated with severe systemic infections, rheumatic fever, or uncomplicated pharyngitis. J Infect Dis 1992;166:374-82.

30. WHO - Epidemic and Pandemic Alert and Response (EPR). Acute respiratory syndrome in China. Available at: http://www.who.int/csr/don/2003_02_11/en/ index.html. Accessed January 8, 2009.

31. WHO - Epidemic and Pandemic Alert and Response (EPR). Acute respiratory syndrome in Hong Kong special administrative region of China/Vietnam. Available at: http://www.who.int/csr/don/2003_03_12/en/index.html.

32. Svoboda T, Henry B, Shulman L, et al. Public health measures to control the spread of the severe acute respiratory syndrome during the outbreak in Toronto. N Engl J Med 2004;350:2352-61.

33. Guan $\mathrm{Y}$, Zhang BJ, He YQ, et al. Isolation and characterization of viruses related to the SARS coronavirus from animals in southern China. Science 2003;302: 276-8.

34. Li W, Shi Z. Bats are natural reservoirs of SARS-like coronaviruses. Science 2005; 310:676-9.

35. Fowler RA, Lapinsky SE, Hallett D, et al. Critically ill patients with severe acute respiratory syndrome. JAMA 2003;290:367-73.

36. Taubenberger JK, Morens DM. 1918 Influenza: the mother of all pandemics. Available at: http://www.cdc.gov/eid/index.htm. Emerging Infect Dis 2006;12: 15-22.

37. Ito T, Couceiro JN, Kelm S, et al. Molecular basis for the generation in pigs of Influenza A viruses with pandemic potential. J Virol 1998;92:7367-73.

38. WHO-epidemic and pandemic alert and response (EPR). Available at: http:// www.who.int/csr/disease/avian_influenza/country/cases_table_2008_09_10/en/ index.html. 